\title{
Three-Dimensional Printing-based Reconstruction of a Maxillary Bone Defect in a Dog Following Tumor Removal
}

\author{
SE EUN KIM ${ }^{1,2^{*}}$, KYUNG MI SHIM $^{1,2^{*}}$, KWANGSIK JANG $^{1,2}$, JIN-HYUNG SHIM ${ }^{3}$ and SEONG SOO KANG ${ }^{1,2}$ \\ ${ }^{1}$ Biomaterial R\&BD Center, Chonnam National University, Gwangju, Republic of Korea; \\ ${ }^{2}$ Department of Veterinary surgery, College of Veterinary Medicine and BK21 Plus Project Team, \\ Chonnam National University, Gwangju, Republic of Korea; \\ ${ }^{3}$ Department of Mechanical Engineering, Korea Polytechnic University, Siheung, Republic of Korea
}

\begin{abstract}
Three-dimensional (3D) printing has been applied extensively not only in human, but also veterinary medicine. However, the technique is still used in the clinical area for a surgical plan or education prior to surgery. Thus, we report a case of reconstruction after tumor removal surgery with the use of a 3D-printed scaffold. A 12-year-old female mixed dog had a left caudal maxillary mass. Based on computed tomography images, a defect was confirmed on the maxillary bone due to the oral mass, and a surgical plan was designed to remove the oral mass and graft the $3 D$ printed scaffold. Customized polycaprolactonel betatracalciumphosphate (PCL/ $\beta-T C P)$ scaffold was fabricated using the micro-extrusion-based $3 D$ printer. In the operation, after the removal of the oral mass, the scaffold was grafted onto the defect site. At follow-up, 8 months after surgery, the result was successful without any special problems in the periodic CT scans and oral examinations. This case is believed to be the first case of reconstruction by using a $3 D$ printed scaffold in the maxillary bone defect, and this $3 D$ printing technique is thought to be very helpful for veterinary patients with bone defects and several other diseases.
\end{abstract}

A 3D printing technology is garnering increased interest, in both industry and medical science. This technology can be used to construct anatomical models for preoperative

This article is freely accessible online.

*These Authors contributed equally to this study.

Correspondence to: Dr. Seong Soo Kang, College of Veterinary Medicine, Chonnam National University, 77, Yongbong-ro, Puk-gu, Gwangju, 61186, Republic of Korea. Tel: +82 625302877, Fax: +82 625302809, e-mail: vetkang@chonnam.ac.kr

Key Words: 3D printing, PCL/ $\beta$-TCP scaffold, oral tumor, dog. planning, training, and education, and has clinical applications in orthopedic, ophthalmic, oral, and maxillofacial surgery $(1,2)$. Researchers and surgeons in the field of oral and maxillofacial surgery have shown particular interest in 3D printing, because the skull, including the maxilla and mandible, has a very complicated structure that is difficult to reconstruct. Adding to the difficulty, many important structures are usually in close proximity to the affected part owing to the anatomical complexity of the region; the uniqueness of individual defects and the possibility of inflammation are additional complications (3). To reconstruct maxillofacial defects, besides maintaining anatomical uniformity and appearance, tissue functions need to be preserved, and 3D printing may be able to address these challenges (4). Three dimensional printing technology enables the creation of complex patient-specific shapes, and enables mixing of various materials to provide optimal strength and function $(5,6)$. In oral and maxillofacial surgery, the main indications for reconstruction are trauma, ablative tumor resection, infection, and congenital/developmental deformities (3). In particular, the increase in the incidence of tumors due to the increase in the number of elderly people has created a need for materials that can replace bone defects caused by tumor removal.

Dogs and cats can also develop oral tumors, that are more likely to be malignant than other tumors. Furthermore, as dogs and cats cannot express the presence of a tumor or pain, a long time may pass before the owner discovers it and seeks treatment. Also for malignant tumors, the surgical margin must be sufficient for complete tumor removal, sometimes creating extensive bone defects, and commonly impacting function or appearance.

To date, the application of 3D printing to this area of veterinary medicine is very limited, here we report a successful reconstruction in a dog with a malignant maxillary squamous cell carcinoma (SCC), using a 3D-printed polycaprolactone /beta-tricalcium phosphate (PCL/ $\beta$-TCP) scaffold. 

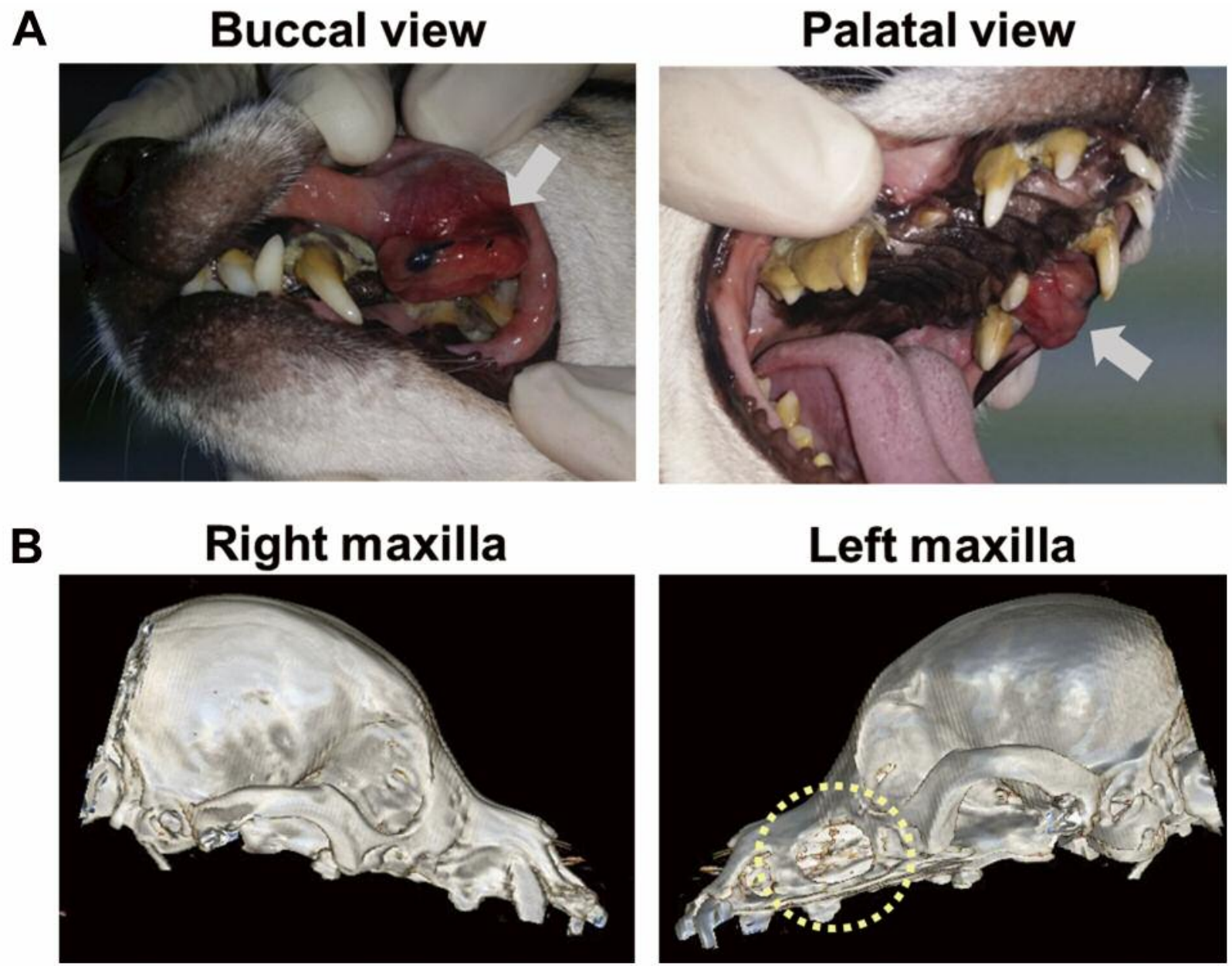

Figure 1. Photographs of the patient's oral tumor in several examinations. (A) In the oral examination, the large mass (arrow) was observed in the left maxilla. (B) Lateral views of $3 D$ volume renderings created from the CT images. Note a large bone defect (circled area) in the left maxilla.

\section{Case Report}

A twelve-year-old female mixed-breed dog presented with left maxillary swelling as well as bleeding around the swelling (Figure 1A). The swelling was identified approximately 7 months prior to visiting of our hospital. Complete blood count, serum biochemistry, and thoracic radiography were performed and showed no specific problems for general anesthesia.

In the surgery, cimetidine $(5 \mathrm{mg} / \mathrm{kg}$, intravenous; i.v., JW Pharmaceutical, Korea), cefazolin $(20 \mathrm{mg} / \mathrm{kg}$, i.v., Hankook Korus Pharm. Co., Ltd., Republic of Korea), enrofloxacin ( $5 \mathrm{mg} / \mathrm{kg}$, SC, Bayer Korea Ltd., Republic of Korea), glycopyrrolate $(0.005 \mathrm{mg} / \mathrm{kg}$, SC, Reyon Pharmaceutical Co., Ltd., Republic of Korea), butorphanol $(0.3 \mathrm{mg} / \mathrm{kg}$, i.v., Myungmoon Pharm. Co., Ltd., Republic of Korea), and midazolam (0.3 mg/kg, i.v., Myungmoon Pharm. Co., Ltd.,) were used for pre-anesthesia, and etomidate $(0.75 \mathrm{mg} / \mathrm{kg}$, i.v., B. Braun Melsungen, Germany) was used for induction of anesthesia. After intubation, anesthesia was maintained with sevoflurane (2.3-4.0\%, Piramal, USA). Ketamine (12 $\mu \mathrm{g} / \mathrm{kg} / \mathrm{min}$, Yuhan Co., Republic of Korea) and tramadol (2 $\mathrm{mg} / \mathrm{kg}$, i.v., Jeil Pharmaceutical Co., Ltd., Republic of Korea) were injected for analgesia.

Under the first round of general anesthesia, an oral examination and dental treatment were performed. In the oral examination, large amounts of dental calculus, gingival recession and gingivitis causing severe periodontal disease were found, and a mass was detected around the left maxillary three and fourth premolar. The mass was firm, pink in color, and $2.0 \mathrm{~cm} \times 3.1 \mathrm{~cm}$ in size; it bordered the gum rim. Dental radiography showed bone lysis around the mass, and the teeth were not in the correct positions. The mass was biopsied and stored in $10 \%$ neutral buffered formalin for 

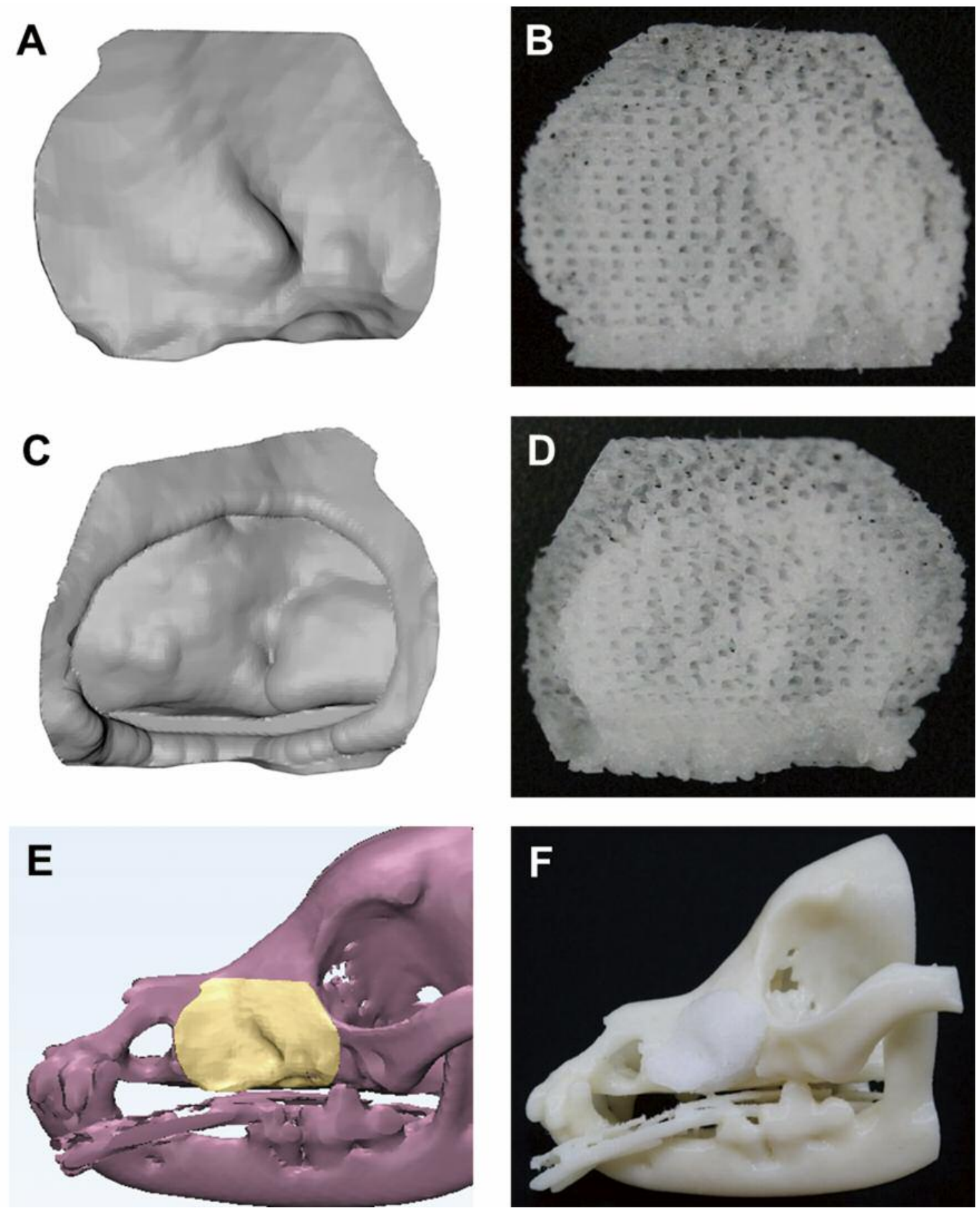

Figure 2. The customized $3 D$ model $(A, C, E)$ and fabricated PCL/ $\beta$-TCP scaffold $(B, D, F)$. Front and back view of scaffold were confirmed (B, $D)$. Fully interconnected pores were confirmed $(B, D)$.

histological analysis. After that, ultrasonic scaling, pumice polishing, and root planning were performed to treat the severe periodontal disease. A fentanyl patch for analgesia (12 $\mu \mathrm{g} / \mathrm{hr}$, Myungmoon Pharm. Co., Ltd., Republic of Korea) was attached for 4 days. Amoxicillin-clavulanic acid $(12.5 \mathrm{mg} / \mathrm{kg}$,
BID, PO, Boryung Co., Ltd., Korea), firocoxib $(2.5 \mathrm{mg} / \mathrm{kg}$, BID, PO, Merial, France), famotidine $(0.5 \mathrm{mg} / \mathrm{kg}$, BID, PO, Taejeon Pharm Wholesale Co., Ltd., Republic of Korea), and milk thistle $(5 \mathrm{mg} / \mathrm{kg}$, BID, PO, Sinil Pharmaceutical Co., Ltd., Republic of Korea) were prescribed for 2 weeks. 

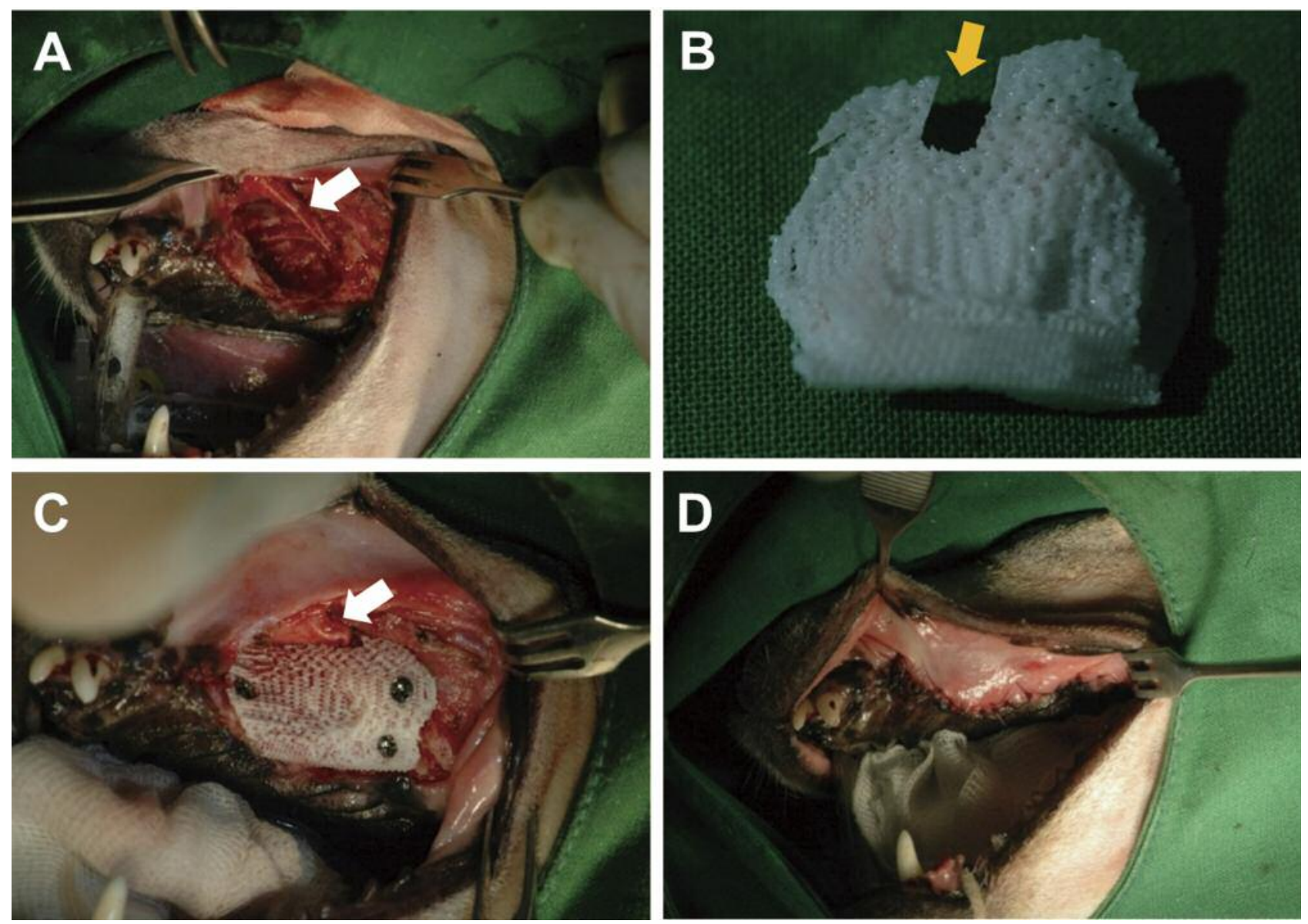

Figure 3. Surgical procedure of reconstruction with 3D-printed PCL/ $\beta$-TCP scaffold after tumor removal in the dog. (A) After the tumor removal, a large bone defect and infraorbital vessels and nerves (white arrow) are seen. (B) For placing the shortened vessels and nerves, a groove (yellow arrow) is made. (C) The 3D-printed scaffold was fixed with mini screws. (D) Photograph after the surgery.

Also, to determine the anatomical state of the skull and investigate whether metastasis had occurred, computed tomography (Siemens Emotion 16; Siemens, Germany) scanning of the dog's entire body was performed with dental treatment. Pre- and post-contrast CT images were acquired at $120 \mathrm{mAs}$ and $130 \mathrm{kV}$ at a $1 \mathrm{~mm}$ slice thickness. Iohexol (880 mgI/kg, i.v., Korea United Pharm., Korea) at a rate of $2 \mathrm{ml} / \mathrm{sec}$ was used for the contrast study. In the presurgical CT scan, the solitary mass was observed at the left caudal maxillary bone and its size was measured at 2.5 $\mathrm{cm} \times 2.7 \mathrm{~cm} \times 2.9 \mathrm{~cm}$. The mass protruded outward, causing maxillary bone lysis, but proliferation or invasion of the ventral nasal cavity were not observed. In the precontrast CT images, the mass showed a homogeneous soft tissue density of 47.5 Hounsfield units (HU), and mild calcification was detected. In the post-contrast CT images, the mass showed a heterogeneous contrast enhancement at 95.4 HU. There was no evidence of the metastasis to lymph nodes or other organs. 3D volume rendering was performed to identify the state of the skull (Figure 1B).

The customized PCL/ $\beta$-TCP scaffold was designed using commercial 3D medical image editing software (Mimics, Materialise, Belgium). The patient's CT image data were saved digitally in the international standard Digital Imaging and Communications in Medicine (DICOM) file format. And, the image data were imported into the Mimics software (Materialise, Belgium), and then CAD model of the skeletal part was automatically generated (Figure 2A, $\mathrm{C}$ and $\mathrm{E})$.

PCL (Evonik Industry, Essen, Germany) and $\beta$-TCP (average diameter $100 \mathrm{~nm}$, Berkeley Advanced Biomaterials Inc., Berkeley, CA, USA) were blended by melting. Chiptype PCL $(4 \mathrm{~g})$ was placed in a glass container and melted at $120^{\circ} \mathrm{C}$ for $15 \mathrm{~min}$. Powdered $\beta$-TCP $(1 \mathrm{~g})$ was added to the molten PCL. The molten PCL and $\beta$-TCP were manually blended for $10 \mathrm{~min}$. 


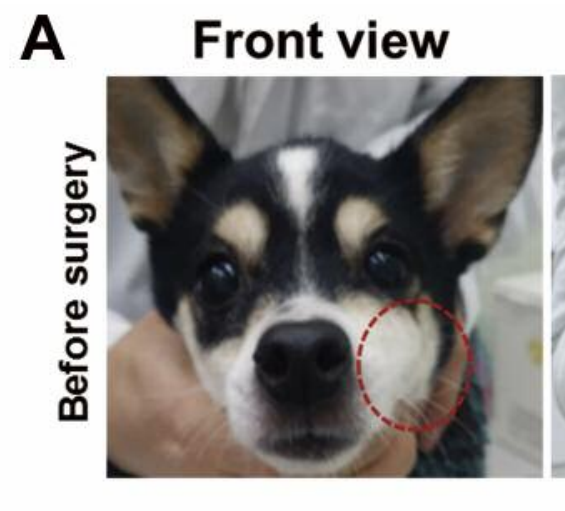

Lateral view

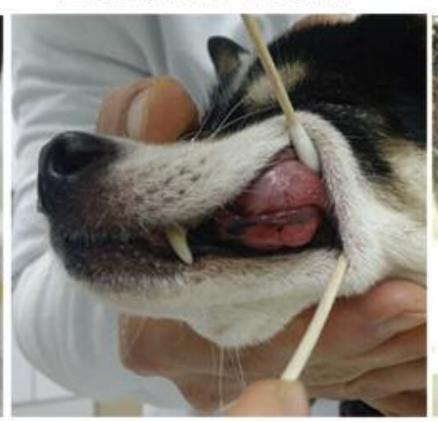

\section{Intraoral view}

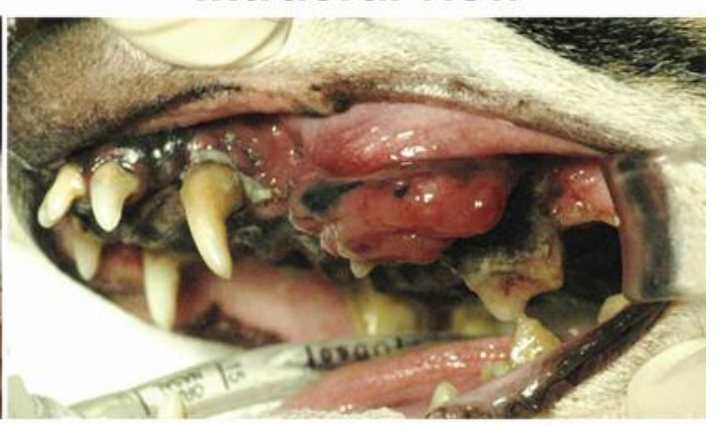

B
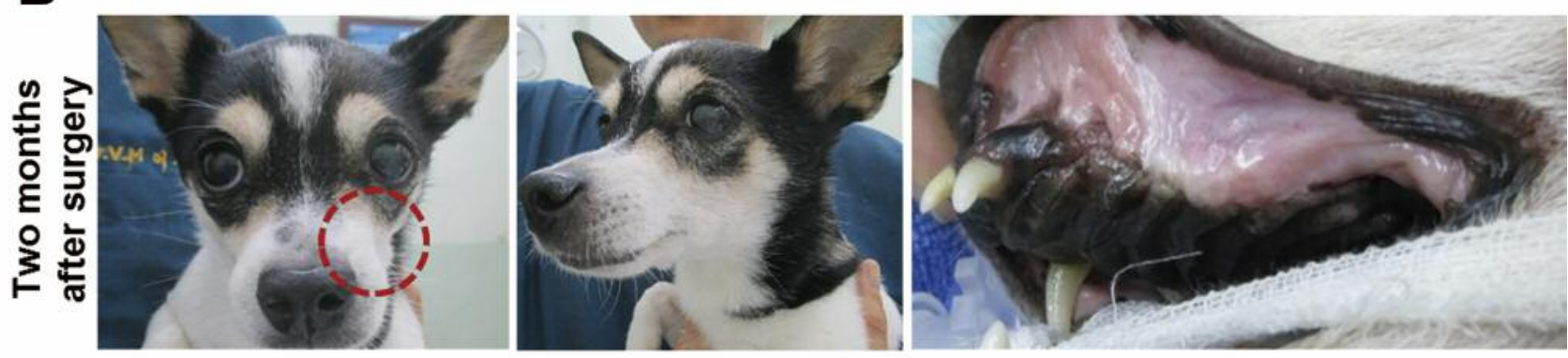

Figure 4. Photographs of the patient before surgery and 2 months after surgery. (A) Before surgery, a large mass in the left maxilla is found. (B) Two months after surgery, the appearance of the left maxilla was same as the opposite side, and the complications related with surgery were not observed.

The blended PCL/ $\beta$-TCP was placed in the $10-\mathrm{ml}$ steel syringe of the microextrusion-based 3D printer (developed inhouse) and the temperature was maintained at $120^{\circ} \mathrm{C}$. The size of the steel nozzle size was $300 \mu \mathrm{m}$, and the pneumatic pressure used for printing was $650 \mathrm{kPa}$. The printing path was generated using CAD/CAM software, and the 3D printing system was operated according to the printing path (Figure 2B, D and F). The line width, pore size, and line height were $300 \mu \mathrm{m}, 500 \mu \mathrm{m}$, and $100 \mu \mathrm{m}$, respectively. The scaffold was designed to have a lattice-type pore architecture. The calculated porosity was approximately $63 \%$ and the pores were fully interconnected. The fabricated scaffold was washed with $70 \%$ ethanol to remove undesirable particles and was sterilized with a $450 \mathrm{~W}$ UV lamp for $3 \mathrm{~h}$.

One month after the first round of anesthesia, the dog was anesthetized again using the same protocol. The mass was surgically removed and stored in $10 \%$ neutral buffered formalin for histological analysis. After trimming the maxillary margin, we applied the 3D printed PCL/ $\beta$-TCP scaffold to the defect (Figure 3A). The scaffold was a plug type, permitting it to be affixed with three 2.0 mini screws (Figure 3C). Because the bone defect caused by the SCC had been present for a long period of time, the infraorbital vessels and nerves had shortened, making it difficult to route them through the infraorbital foramen of the engineered scaffold; we therefore created a groove in the upper part of the scaffold for the blood vessels (Figure 3B). And, the reconstructed defect was closed with the mucosa using 5-0 poliglecaprone 25 (Monocryl, Ethicon, USA) (Figure 3D). The same analgesics and antibiotics used following the last CT scan were prescribed.

CT scanning of the whole body was performed immediately and 2 months after the surgery (Figure 4). CT scanning immediately after surgery revealed that the oral mass was completely removed and the 3D printed scaffold was affixed using screws. It was confirmed that the two screws near the orbit and the one screw near the nasal bone were securely affixed. Two months following the surgery, another CT scan was performed. In CT scan images, the 3D printed scaffold was confirmed to be correctly located and affixed by the screws. New bone and soft tissue were observed to be forming inside and around the graft area. Liquid and soft tissue-like findings were observed in the nasal cavity on the opposite site; however, these findings were determined to have been caused by the pre-existing periodontal disease.

After the surgery, periodic examinations were conducted during 8 months. Four days after the surgery, the surgical site appeared healthy, without complications such as 


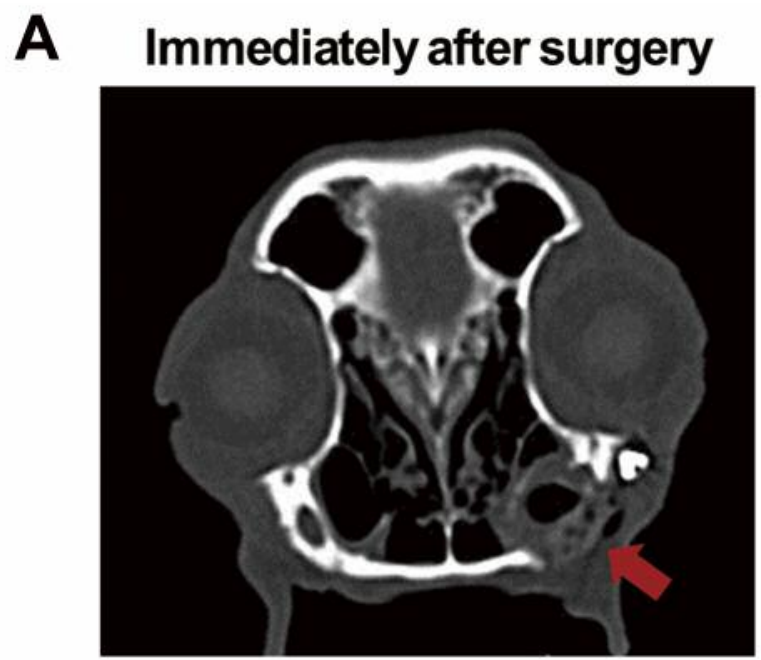

\section{B Two months after surgery}
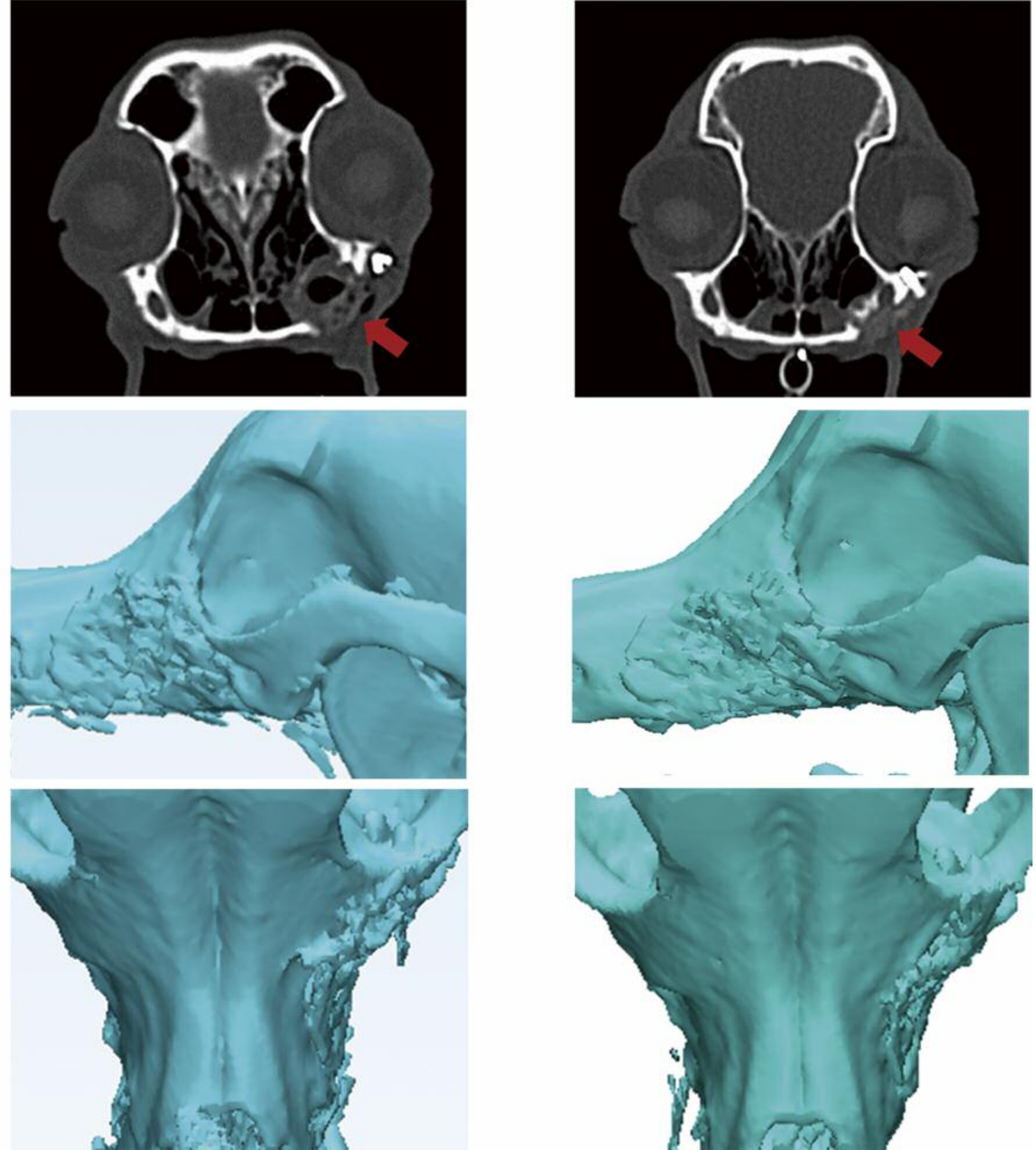

Figure 5. Coronal view and $3 D$ volume rendering CT images of the patient immediately and 2 months after surgery. (A) Immediately after surgery, grafted $3 D$ printed PCL/ $\beta$-TCP scaffold (arrow) was placed and shown as a porous material. (B) Two months after surgery, newly-formed bone under the scaffold (arrow) was observed.

bleeding, swelling, or dehiscence. At 2 and 7 months after the surgery, the maxillary appearance was restored, and appeared the same as the healthy side, without complications related to the surgery or the implanted $3 \mathrm{D}$ printed scaffold (Figure 5). At 8 months after the surgery, we conducted a follow-up visit over the phone because the owner was unable to visit our hospital, and found no complications. 


\section{Discussion}

In dogs, tumors of the oral cavity represent $5-7 \%$ of all diagnosed tumors (7). The most common oral tumors in dogs include malignant melanoma, SCC, fibrosarcoma, osteosarcoma, acanthomatous ameloblastoma, and peripheral odontogenic fibromas (8). Among these, SCC is the second most common oral cavity tumor, and it occurs in the gingiva, tonsils, oral mucosa, lip, and palate (9). SCC produces dental disruption and bone invasion in the lesion. When the SCC is positioned in the rostral area, metastasis is rare, but tumors in the caudal area of the oral cavity show a higher rate of metastasis (10).

Treatments for SCC include medication, chemotherapy, surgical removal, and radiation therapy. Because canine SCC tumor cells overexpress COX-2, medications that inhibit COX-2, such as piroxicam and meloxicam, are used to treat it (11). Although piroxicam slows the progression of oral SCC, it cannot completely remove SCC, and can only be used as an adjuvant therapy (12). Canine SCC can be treated with radiation therapy, and this treatment can be applied when surgery is not possible due to tumor size or location, or if the surgical margin is incomplete (13). However, few radiation facilities are available for veterinary patients.

The optimal treatment for SCC is complete removal of the mass. The local recurrence rate of SCC is $17.2 \%$ and the metastatic rate is $3.4 \%$; the recurrence rate increases if the tumor is not removed completely and a surgical margin of at least $1 \mathrm{~cm}$ is required for complete removal $(8,14)$. In particular, if a bone lesion is involved, bone margins and ablation should be applied (15). However, these treatments cause complications including swelling, partial dehiscence, or an appearance that is not acceptable to owners. In this case, after removing the mass and trimming the bones, the most pressing problem is dehiscence, because the large defects created by surgery must be covered with mucosa only.

During a maxillectomy or mandibulectomy, the primary goal is ensuring that the surgical margins are kept clean, but preserving normal function and appearance after surgery should also be considered. Thus, in this case we decided to reconstruct the caudal maxillary bone defects with a $3 \mathrm{D}$ printed scaffold after tumor removal surgery.

The 3D printed composite scaffold used here was designed to optimize mechanical strength and biocompatibility, and is also the most popular type of scaffold for bone regeneration. Composite scaffolds are usually printed using synthetic polymers such as polylactic acid (PLA), polyglycolic acid (PGA), polylactic-polyglycolic acid (PLGA), polyethylene glycol (PEG), and PCL, combined with hydroxyapatite (HA) or $\beta$-TCP (16-18). In this case, we used PCL and $\beta$-TCP.

PCL is an aliphatic polyester with good mechanical properties and a long resorption time, but poor osteoconductivity (19). The 3D printed scaffolds using PCL have been approved by the Food and Drug Administration
(FDA) for bone tissue engineering (20). And, $\beta$-TCP is an inorganic matrix that is commonly used for bioceramics. It has several advantages over HA, including the ability to control pore size and manufacture a suitable morphology; however, because it has low mechanical strength, combination with other materials is recommended (21). Thus, to compensate for these shortcomings, we used a combination of these two materials for printing.

To the authors' knowledge, this is the first report of a reconstruction using a $3 \mathrm{D}$ printed scaffold in maxilla in Veterinary Dentistry, with the exception of experimental reconstructions. The surgery was successful, without adverse effects such as discomfort, pain, or dehiscence.

Advances in medical technology have also led to increased longevity in dogs and cats, causing an increase in tumors of the bone, lung, liver, intestines, and other organs. For complete removal of tumors and prevention of recurrence, a surgical margin of $1-5 \mathrm{~cm}$ is required, depending on tumor type, regardless of the weight and size of the veterinary patient. Thus, in small dogs and cats it is very difficult to reconstruct defects, and the few alternatives have several limitations. Thus, 3D printing technology may provide a solution for this problem. However, to date most applications of 3D printing have been educational, such as creation of anatomical models, and its clinical application has been limited to planning prior to surgery (10).

3D printing technology is now starting to be used in veterinary clinics, especially for implants for grafting into the body. In addition, scaffolds loaded with cells or growth factors, or those containing collagen or decellularized extracellular matrix, that can improve tissue regeneration, can be applied in veterinary medicine earlier than in human medicine. Thus, this technology shows promise for use in treating disease, improving the quality of life not only for companion animals, but also for their owners.

\section{Acknowledgements}

This research was supported by a grant from the Korea Health Technology R\&D Project through the Korea Health Industry Development Institute (KHIDI), funded by the Ministry of Health \& Welfare (grant number: HI14C3309) and Basic Science Research Program through the National Research Foundation of Korea (NRF), funded by the Ministry of Education (2017R1A6A3A11030650).

\section{References}

1 Martelli N, Serrano C, van den Brink H, Pineau J, Prognon P, Borget I and El Batti S: Advantages and disadvantages of 3dimensional printing in surgery: A systematic review. Surgery 159(6): 1485-1500, 2016.

2 Hoang D, Perrault D, Stevanovic M and Ghiassi A: Surgical applications of three-dimensional printing: A review of the current literature \& how to get started. Ann Transl Med 4(23): 456, 2016. 
3 Parthasarathy $\mathrm{J}$ : $3 \mathrm{~d}$ modeling, custom implants and its future perspectives in craniofacial surgery. Ann Maxillofac Surg 4(1): 9, 2014.

4 Bhumiratana S and Vunjak-Novakovic G: Concise review: Personalized human bone grafts for reconstructing head and face. Stem Cells Transl Med 1(1): 64-69, 2012.

5 Kern R: 3-D printed implants hit the market, pave the way for more personalized devices. The Gray Sheet 39, 2013.

6 Rengier F, Mehndiratta A, von Tengg-Kobligk H, Zechmann CM, Unterhinninghofen R, Kauczor H-U and Giesel FL: 3d printing based on imaging data: Review of medical applications. Int J Comput Assist Radiol Surg 5(4): 335-341, 2010.

7 Frew D and Dobson J: Radiological assessment of 50 cases of incisive or maxillary neoplasia in the dog. J Small Anim Pract 33(1): 11-18, 1992.

8 Sarowitz B, Davis G and Kim S: Outcome and prognostic factors following curative-intent surgery for oral tumours in dogs: 234 cases (2004 to 2014). J Small Anim Pract 58(3): 146$153,2017$.

9 Dorn C and Priester W: Epidemiologic analysis of oral and pharyngeal cancer in dogs, cats, horses, and cattle. J Am Vet Med Assoc 169(11): 1202-1206, 1976

10 Carpenter L, Withrow S, Powers B, Ogilvie G, Schwarz P, Straw $\mathrm{R}$, LaRue $\mathrm{S}$ and Berg J: Squamous cell carcinoma of the tongue in 10 dogs. J Am Anim Hosp Assoc, 1993.

11 Verhaert L: Oral proliferative lesions in the dog and cat. EUR J Companion Anim Pract 20(3): 1-13, 2010.

12 Schmidt BR, Glickman NW, DeNicola DB, Gortari AEd and Knapp DW: Evaluation of piroxicam for the treatment of oral squamous cell carcinoma in dogs. J Am Vet Med Assoc 218(11): 1783-1786, 2001.

13 Grier CK and Mayer MN: Radiation therapy of canine nontonsillar squamous cell carcinoma. Can Vet J 48(11): 1189, 2007.

14 Kosovsky JK, Matthiesen DT, Marretta S and Patnaik AK: Results of partial mandibulectomy for the treatment of oral tumors in 142 dogs. Vet Surg 20(6): 397-401, 1991.
15 Salisbury SK, Richardson DC and LANTZ GC: Partial maxillectomy and premaxillectomy in the treatment of oral neoplasia in the dog and cat. Vet Surg 15(1): 16-26, 1986.

16 Luangphakdy V, Walker E, Shinohara K, Pan H, Hefferan T, Bauer TW, Stockdale L, Saini S, Dadsetan M and Runge MB: Evaluation of osteoconductive scaffolds in the canine femoral multi-defect model. Tissue Eng Part A 19(5-6): 634-648, 2013.

17 Carrel JP, Wiskott A, Scherrer S and Durual S: Large bone vertical augmentation using a three-dimensional printed tcp/ha bone graft: A pilot study in dog mandible. Clin Implant Dent Relat Res 18(6): 1183-1192, 2016.

18 Shim J-H, Kim SE, Park JY, Kundu J, Kim SW, Kang SS and Cho D-W: Three-dimensional printing of rhbmp-2-loaded scaffolds with long-term delivery for enhanced bone regeneration in a rabbit diaphyseal defect. Tissue Eng Part A 20(13-14): 1980-1992, 2014.

19 Rezwan K, Chen Q, Blaker J and Boccaccini AR: Biodegradable and bioactive porous polymer/inorganic composite scaffolds for bone tissue engineering. Biomaterials 27(18): 3413-3431, 2006.

20 Iacobellis C, Berizzi A and Aldegheri R: Bone transport using the ilizarov method: A review of complications in 100 consecutive cases. Strategies Trauma Limb Reconstr 5(1): 1722, 2010

21 Polo-Corrales L, Latorre-Esteves $\mathrm{M}$ and Ramirez-Vick JE: Scaffold design for bone regeneration. J Nanosci Nanotechnol 14(1): 15-56, 2014. 